\title{
Rehabilitating human oocytes by polar body transplantation
}

\author{
David F. Albertini ${ }^{1}$
}

Published online: 8 May 2017

(C) Springer Science+Business Media New York 2017

If only we knew what Mother Nature's defining secrets were for those gametes endowed with healthy baby-making properties! As the search for reliable biomarkers of gamete and embryo quality continues unabated, there is no escaping the fact that many patients never reach embryo transfer for any of a number of factors. Whether under the watchful eye of the embryologist, or discretionary algorithms sifting through a time lapse sequence, or the dreaded "aneuploidy" verdict after PGS, many of the embryos rejected as a result of our current assessment methods acquire their ultimate fate for any number of poorly understood reasons.

Among the suspects of historical significance has been the incidence and extent of fragmentation during the early cleavage stages of development. And as we will see below, it is not uncommon to encounter patients who all too uniformly, and repeatedly, generate fragmented embryos. If, as many believe, the underlying defects reside somewhere within the oocyte cytoplasm, then would this condition not indicate yet another intervention relying in the final analysis on a reconstruction project similar to the seasoned concept of cytoplasmic transfer?

One of the more remarkable feats that an oocyte accomplishes during its quest to achieve nuclear and cytoplasmic maturity is emission and abscission of the polar bodies. As shown on our cover this month, somehow in completing the process of meiosis, the female gamete has figured out how to dispose of three quarters of its genome, in the first and second polar bodies combined, and yet retain the vast majority of cytoplasm with its vital contents to be reserved for the

David F. Albertini

dalbertini@thechr.com

1 The Center for Human Reproduction, New York, NY, USA embryo. This form of asymmetric cytokinesis guarantees getting a glimpse of chromosome balance in the resulting oocyte when the polar body is removed and analyzed for its genetic composition-as was originally demonstrated and put into practice by the late Yuri Verlinsky [1]. While being neglected for the longest time, these castoff genomes have been explored and exploited recently as much more than sentinels of oocyte ploidy as many investigators have sought and achieved insights into the parent specific - especially maternal - contributions to the origins and later impact of meiotic transgressions on the fate of the resulting conceptus [2-4].

But could these genomic castoffs be used for a clinical purpose? In other words, might polar body genomes be repurposed in another context for other therapeutic uses? It is in this context that the studies from Zhang and colleagues appear this month in JARG place yet another plausible intervention on the ART menu that should appear specifically under the heading of Gamete Rehabilitation [4].

Ge Lin and colleagues first recognized the severity of fragmentation exhibited by the embryos from one of their patients. Prompted by this case, they initiated a series of experiments using immature M1 stage oocytes that after overnight in vitro maturation produced 104 mature oocytes out of the 298 oocytes they cultured. With this material, as described in their schematic in Figure 2, polar body biopsy, spindle enucleation, and cell fusion techniques were undertaken to produce zygotes before or after ICSI and reconstructed oocytes were monitored for further development. In some experiments, vitrified first polar bodies (PB1) were used in reconstructions, and although the numbers are small, they report that with respect to normal fertilization and blastocyst formation, there was no difference between using fresh or vitrified PBs.

Over and above the confluence of interventional ARTs in making a proof-of-principle statement (albeit with a limited number of samples), the real significance for provocative 
publications like this is whether there is a legitimate clinical context into which a procedure like PB transplantation would fit. Here, as Zhang et al. show, emerges a promising strategy worthy of further study. Using cryopreserved PB1s from the patient mentioned earlier, and enucleated donor oocytes, the embryos produced after PB1 transplantation exhibited less fragmentation and better progression to the blastocyst stage. But do not hold your breath for too long! Of the 15 donor oocytes reconstructed with the patient's PBs, only seven showed signs of normal fertilization and first cleavage and four reached the blastocyst stage, of which three were diploid and one aneuploid.

Clearly, there is a long way to go before PB transplantation makes headway clinically or ethically. Moreover, there are some serious drawbacks in this work. For example, the actual genotype of the experimental and case-related embryos produced by PB transplantation was never determined. This is a must if any level of certainty is to be rendered as to the corrective capability of donor cytoplasts. In addition, there will be clear consequences depending on the use of genomes from either PB1s or PB2s, over and above the obvious distinctions in the nature of the chromosomes that are received by the cytoplast and how these respond to the intrinsic cell cycle state of the recipient oocyte. Finally, using in vitro matured oocytes may only reinforce, if not amplify, developmental deficiencies that could appear well beyond the blastocyst if reconstructed oocytes were ever to be transferred.

In closing this most recent chapter of emerging rehabilitation projects for continued cogitation, we should be mindful of the checks and balances we are obliged to implement on the pathway to new and modified human ARTs. Animal studies always should play an important role in attesting to safety and efficiency on the pathway to clinical trials. And as it turns out, studies using mouse oocytes have recently been published by
Yamada and Egli [5]. Using spindle exchange rather than PB transplantation, they show that genomes recovered from aged oocytes and placed into "young" cytoplasts recover full developmental competence as assayed by blastocyst formation (from parthenotes) or production of term pups when IVF and transfer of reconstructed embryos was used. Beneath the success of such procedures in animal models, the authors decipher a mechanistic explanation for the loss of oocyte quality in aged oocytes based upon a thorough evaluation of cytokinesis and the involvement of cell cycle factors.

We at JARG look forward to keeping our readership informed with regard to the latest advances in reproductive genetics and welcome your feedback on both our coverage of these topics and your suggestions for future issues.

\section{References}

1. Verlinsky Y, Ginsberg N, Lifchez A, Valle J, Moise J, Strom CM. Analysis of the first polar body: preconception genetic diagnosis. Hum Reprod. 1990;5(7):826-9.

2. Fragouli E, Alfarawati S, Goodall NN, Sanchez-Garcia JF, Colls P, Wells D. The cytogenetics of polar bodies: insights into female meiosis and the diagnosis of aneuploidy. Mol Hum Reprod. 2011;17(5):286-95.

3. Christopikou D, Tsorva E, Economou K, Shelley P, Davies S, Mastrominas M, et al. Polar body analysis by array comparative genomic hybridization accurately predicts aneuploidies of maternal meiotic origin in cleavage stage embryos of women of advanced maternal age. Hum Reprod. 2013;28(5):1426-34.

4. Zhang SP, Lu CF, Gong F, Xie PY, Hu L, Zhang SJ, et al. Polar body transfer restores the developmental potential of oocytes to blastocyst stage in a case of repeated embryo fragmentation. J Assist Reprod Genet. 2017. doi:10.1007/s10815-017-0881-y.

5. Yamada M, Egli D. Genome transfer prevents fragmentation and restores developmental potential of developmentally compromised postovulatory aged mouse oocytes. Stem Cell Reports. 2017. doi:10. 1016/j.stemcr.2017.01.020. 